\title{
Insights into the physiology of Methylotenera mobilis as revealed by metagenome-based shotgun proteomic analysis
}

\section{Correspondence \\ Ludmila Chistoserdova \\ milachis@u.washington.edu}

Received 9 October 2008

Revised 2 January 2009

Accepted 5 January 2009

\author{
Gundula Bosch, ${ }^{1}$ Tiansong Wang, ${ }^{2}$ Ekaterina Latypova, ${ }^{1}$ \\ Marina G. Kalyuzhnaya, ${ }^{2}$ Murray Hackett ${ }^{1}$ and Ludmila Chistoserdova ${ }^{1}$ \\ ${ }^{1}$ Department of Chemical Engineering, University of Washington, Seattle, WA 98195, USA \\ ${ }^{2}$ Department of Microbiology, University of Washington, Seattle, WA 98195, USA
}

\section{INTRODUCTION}

The number of microbial species whose genomes have been completely sequenced is steadily growing; however, these organisms still represent only a fraction of cultivated microbes. These in turn represent only a fraction of existent microbes (Rappé \& Giovannoni, 2003), thus making the overall genomic coverage of microbial diversity minuscule. The recent surge in metagenomic sequencing, and downstream applications such as metatranscriptomics and metaproteomics (Ram et al., 2005; Denef et al., 2007; Frias-Lopez et al., 2008; Gilbert et al., 2008), are transforming the field of microbiology. However, due to the novelty of these approaches and to their inherent shortcomings such as low sequence coverage and low sampling depth (Tringe et al., 2005; Rusch et al., 2007), the utility and the potential applications of these datasets remain poorly explored. Recently, applicability of highquality genomic information in the identification of peptides between related environmental strains has been explored, using a probability-based model and a random mutation simulation model (Denef et al., 2007). Here we explore a reversed approach, using a recently generated metagenomic dataset representing multiple strains of a single bacterial species, M. mobilis (Kalyuzhnaya et al.,

Abbreviations: GS/GOGAT, glutamine synthetase/glutamate synthase; RMP, ribulose monophosphate.

A supplementary table is available with the online version of this paper. 2008b), for protein detection in the type strain of Methylotenera mobilis, JLW8, a laboratory-cultivated strain.

M. mobilis JLW8 is the only formally described representative of Methylotenera, a new genus within the family Methylophilaceae (Kalyuzhnaya et al., 2006). All known members of this family are obligate methylotrophs, i.e. they are specialized in degradation of organic compounds containing no carbon-carbon bonds ( $\mathrm{C}_{1}$ compounds), most prominently methanol and methylamine (Lidstrom, 2006). Based on culture-independent surveys, Methylotenera species appear to be ubiquitous, having been detected in marine, freshwater, acid mine drainage, polluted soil, sedimentary rock and glacier environments (according to the entries in the non-redundant database, http://www.ncbi.nlm.nih.gov/), consistent with an important role in global carbon cycling. However, the exact function of these bacteria remains unknown. M. mobilis JLW8 was isolated from a $63 \mathrm{~m}$ deep sampling site in Lake Washington sediment (Kalyuzhnaya et al., 2006). Samples used for stable isotope probing-based enrichment of methylotrophic communities that were also used for metagenomic sequencing were collected from the same site, but at a later date (Kalyuzhnaya et al., 2008b). From metagenomic analysis conducted on these samples, the methylamine microcosm was dominated by $M$. mobilis strains, which allowed for extracting a composite genome of M. mobilis and conducting metabolic reconstruction for this species (Kalyuzhnaya et al., 2008b). However, the dominant strains of $M$. mobilis represented in the 
metagenome appear to be distinct from M. mobilis JLW8, based on $16 \mathrm{~S}$ rRNA sequence comparisons. The study undertaken here pursued three main objectives: (1) to validate the metagenome-based sequence assembly and binning of $M$. mobilis sequences conducted in the previous study, (2) to test whether this metagenomic sequence provides a quality protein database for peptide identification in a strain without perfect matches in the metagenome and test the limitation of such a database, and (3) to obtain new insights into the physiology of $M$. mobilis.

\section{METHODS}

Cultivation and harvesting. M. mobilis JLW8 was grown on methylamine, the only substrate identified so far that supports its growth in the laboratory, essentially as described previously (Kalyuzhnaya et al., 2006). Per biological replicate, cells from a 21 culture $\left(\mathrm{OD}_{600} 0.2\right)$ were harvested by centrifugation at $4500 \mathrm{~g}$ for $8 \mathrm{~min}$ at $4{ }^{\circ} \mathrm{C}$, briefly washed with cooled $20 \mathrm{mM}$ Tris/ $\mathrm{HCl} \mathrm{pH} 8$, flash-frozen in liquid nitrogen and stored at $-80{ }^{\circ} \mathrm{C}$.

Protein extraction and digestion. Frozen cell pellets were resuspended in $500 \mu \mathrm{l}$ hot resuspension buffer $(20 \mathrm{mM}$ Tris/ $\mathrm{HCl}$ $\mathrm{pH} 8.0,5 \mathrm{mM}$ DTT) and lysed by boiling for $2 \mathrm{~min}$ in a water bath, followed by cooling on ice for $10 \mathrm{~min}$. For nucleic acid digestion, 10 units benzonase nuclease (Roche) was added after adjusting the suspension to $2 \mathrm{mM} \mathrm{MgCl}_{2}$ (final concentration). After $15 \mathrm{~min}$ incubation at room temperature, $200 \mu \mathrm{l}$ of ethanol- and bufferwashed glass beads $(150 \mu \mathrm{m})$ was added, the sample volume was adjusted to $1.5 \mathrm{ml}$ with resuspension buffer, and bead beating was performed for $4 \mathrm{~min}$ at 48 r.p.m. in a MiniBeadBeater (BioSpec Products). Total protein concentration was determined by Bradford protein assay (Bio-Rad); this was approximately $1 \mathrm{mg} \mathrm{ml}^{-1}$. The homogenate was lyophilized to dryness. After resuspension in $500 \mu \mathrm{l}$ digestion buffer (2 $\mathrm{M}$ urea, $5 \%$ acetonitrile, $5 \mathrm{mM}$ dithiothreitol, $0.1 \%$ Rapigest), the sample was digested as previously described (Bosch et al., 2008).

HPLC pre-fractionation and linear ion trap mass spectrometry. The soluble fraction after digestion was lyophilized to a volume of approximately $150 \mu \mathrm{l}$ and centrifuged for $10 \mathrm{~min}$ at 14000 r.p.m. in a tabletop centrifuge (Eppendorf). A 10-20 $\mu$ l sample of the supernatant was applied to a PLRP-S reversed-phase column $(2.1 \mathrm{~mm}$ i.d. $\times 150 \mathrm{~mm}, 300 \AA$, $5 \mu \mathrm{m}$; Polymer Laboratories) with mobile phases of $0.1 \%$ trifluoroacetic acid in $\mathrm{H}_{2} \mathrm{O}$ and acetonitrile. Peptides were eluted at $0.2 \mathrm{ml} \mathrm{min}^{-1}$ with a gradient of 2-60\% acetonitrile in $60 \mathrm{~min}$ and $60-90 \%$ acetonitrile in $20 \mathrm{~min}$ and collected as five separate fractions. The fractions were each lyophilized to $20 \mu \mathrm{l}$, and after reconstitution to volumes of $120 \mu \mathrm{l}$ with acetic acid and acetonitrile at final concentrations of $0.5 \%$ and $5 \%(\mathrm{v} / \mathrm{v})$, respectively, subjected to LC/LC-MS/MS (two-dimensional capillary HPLC/linear ion trap tandem mass spectrometry) analysis as previously described (Bosch et al., 2008).

SEQUEST database searching, DTAselect filtering and estimation of random false positive identification rate. Using the SEQUEST algorithm, raw data were searched against a concatenated FASTA database that consisted of polypeptide sequences derived from the M. mobilis composite genome (Kalyuzhnaya et al., 2008b), the human subset of the non-redundant database (nrdb) depleted of all virus sequences, and reversed sequence (decoy) versions of both databases appended. The concatenated database comprised a total of 214148 protein sequences, 12951 of which represented the M. mobilis specific meta-database. The search results were filtered in DTAselect applying the parameters described by Bosch et al. (2008). Sequest $\Delta \mathrm{Cn} / \mathrm{Xcorr}$ values of $0.08 / 1.9,0.08 / 2.2$ and $0.08 / 3.3$ were used for singly, doubly and triply charged peptides, respectively. Three peptides unique to a particular ORF were required for positive identification. Random false positive identifications were determined at the peptide level as a ratio of two times the number of reversed peptide identifications in the concatenated database to the total number of identified peptides (Elias \& Gygi, 2007). By this means, the qualitative random false positive rate was estimated to be $1.5 \%$ for the first biological replicate and $2.1 \%$ for the second biological replicate.

Data processing and normalization. The in-house methods developed for estimating protein abundances from spectral counting values have been described by Xia et al. $(2007 \mathrm{a}, \mathrm{b})$. In order to normalize the two biological replicates, the total sum of the average spectral counts for the second biological replicate was divided by the total sum of the average spectral counts for the first biological replicate, resulting in a normalization factor of 1.077. Each average spectral count value in the first biological replicate was multiplied by this normalization factor.

Reproducibility of biological replicates. Linear regression analysis demonstrated good data reproducibility for the two biological replicates $(R=0.86$ for the 1924 proteins common to both replicates; data not shown).

DNA amplification, sequencing and analysis. DNA of $M$. mobilis JLW8 was isolated using a QIAamp DNA minikit (Qiagen). Semirandomly selected fragments of DNA, as shown in Table 1, were amplified by PCR, cloned and sequenced essentially as described by Kalyuzhnaya et al. (2008a). Oligonucleotide primers for amplification were designed based on the metagenomic sequence, as follows. First, a gene to be amplified was chosen (genes involved in methylotrophy, central pathways or hypothetical genes). All the homologues representing different $M$. mobilis strains in the metagenome were then aligned using tools available as part of the Joint Genome Institute IMG/M package (http://img.jgi.doe.gov/cgi-bin $/ \mathrm{m} / \mathrm{main}$.cgi), and consensus sequences were chosen with no or few mismatches.

Peptide mass predictions. Peptide mass predictions were done using the PeptideMass program available at the ExPASy Server (Gasteiger et al., 2005).

Enzyme assays. Cells were grown on methylamine as above, to $\mathrm{OD}_{600} 0.8$, pelleted by centrifugation, as above, resuspended in Tris/ $\mathrm{HCl} \mathrm{pH} 8.0$ buffer and disrupted by passage through a French pressure cell at $1.2 \times 10^{8} \mathrm{~Pa}$. Cell extracts were centrifuged at 14000 r.p.m. for $25 \mathrm{~min}$ at $4{ }^{\circ} \mathrm{C}$ to remove cell debris. The activity of glutamine synthetase was assayed as described by Murrell \& Dalton (1983), the activity of glutamate synthase was assayed as described by Bravo \& Mora (1988), and the activity of glutamate dehydrogenase was assayed as described by Yarrison et al. (1972).

\section{RESULTS AND DISCUSSION}

\section{Protein divergence between M. mobilis JLW8 and environmental $M$. mobilis}

In the composite genome of $M$. mobilis that is part of the recently described metagenomic dataset (Kalyuzhnaya et al., 2008b), there are three matches to the 16S rRNA gene of M. mobilis JLW8. One of these is a small fragment (291 nt) that seems to represent a very conserved part of 
Table 1. Comparison of proteins translated from DNA fragments of M. mobilis JLW8 with homologous proteins translated from the composite genome

ID, identity. Proteins (sequences of oligonucleotide primers used for PCR amplification are shown in parentheses, first forward and then reverse): MtdB, methylene $\mathrm{H}_{4} \mathrm{MPT}$ dehydrogenase (atggaaaaagctagcattt, ttaagagtgaacttttaat); Fae, formaldehyde activating enzyme (gtgtttggtttaaccatc, cagcacaacgcggtgttg); FdhA, large subunit of formate dehydrogenase (gattgccttacttgtgccac, ctgaatacgggttaactcg [1]; atgaagattgggaagtcacg, cgcttcactaaatgtcttg [2]); Fdh4A, large subunit of formate dehydrogenase (gaagactccatgagtatggtg, gattatcaccgggatagac); Mdh, malate dehydrogenase (gagattgactgcgattgatg, aaggttaccggtggtaatccagtgcgcg); AceE, component E1 of pyruvate dehydrogenase complex (gtggcttgatgctttaatg, ctaatgattcaggctcatc [1]; ctgcaaccattccaaattg, cggcttcagccactttagc [2]); GltA, GltB, large and small subunits of glutamate synthase (cgtgattcgtaaacgtcttg, ccatcattacgatgtcatc; acaagatccagaattgcgtaaac, gcgcctgagttacgcacacag); MauN, polyferredoxin involved in methylamine oxidation (ctgatcggggcgccattgtg, tatcacgccccttgctccgc); PrpB, methylisocitrate lyase (cggtgccatcaacgcttac, cgcagagagtggatatagc); TR, predicted transcriptional regulator (gagattgactgcgattgatg, ggtggtaatccagtgcgcg); Hp, hypothetical protein (tgcaaccattccaaattg, ggcttcagccactttagc [1]; gttattaagccataccatc, gagggtgattggtcgcag [2]).

\begin{tabular}{|c|c|c|c|c|c|}
\hline Protein & Length (aa) & No. of matches $\dagger$ & Coverage range $(x)$ & ID range $(\%)$ & $\begin{array}{l}\text { ID for most covered } \\
\text { protein }(\%)\end{array}$ \\
\hline $\operatorname{MtdB}^{\star}$ & 307 & $2(2)$ & $2.8-3.9$ & 93 & 93 \\
\hline Fdh4A & 241 & $6(3)$ & $1.4-4.3$ & $90-91$ & 91 \\
\hline Mdh & 224 & $6(3)$ & $1.5-3.8$ & $92-96$ & 93 \\
\hline AceE $[1] \ddagger$ & 224 & $7(3)$ & $1.5-3.1$ & $95-99$ & 99 \\
\hline FdhA [1]末 & 222 & $6(1)$ & $1.5-3.8$ & $94-96$ & 96 \\
\hline FdhA [2]末 & 198 & $10(1)$ & $1.2-4.2$ & $95-100$ & 97 \\
\hline GltA & 197 & $8(1)$ & $1.2-3.6$ & $95-100$ & 100 \\
\hline GltB & 220 & $8(1)$ & $1.6-3.6$ & $94-97$ & 94 \\
\hline MauN & 180 & $4(3)$ & $1.6-5.1$ & $94-96$ & 95 \\
\hline PrpB & 171 & $4(3)$ & $1.4-3.2$ & $98-99$ & 98 \\
\hline
\end{tabular}

${ }^{\star}$ Complete sequences of MtdB and Fae were obtained. All other sequences are partial.

$\dagger$ The number of full-length sequences is shown in parentheses.

¥In the cases of AceE and FdhA, two different fragments of the same protein were obtained. In the case of HP, two different proteins are discussed.

the gene, with $100 \%$ homology to the M. mobilis JLW8 16S rRNA gene, at low sequence coverage $(1.8 \times$; Kalyuzhnaya et al., 2008b). Two others are nearly complete genes with 97 and $98 \%$ homology, respectively, present at much higher coverage $(13.2 \times$ and $20.4 \times$, respectively). Typically, the level of divergence for protein-coding genes between different strains is higher than for $16 \mathrm{~S}$ rRNA genes (Konstantinidis \& Tiedje, 2005). To obtain an estimate of divergence between proteins of M. mobilis JLW8 and proteins of $M$. mobilis represented in the metagenome, we amplified 15 DNA fragments, as described in Methods, translated polypeptide sequences from these, and compared them to the metagenome-based M. mobilis protein database. Each query peptide had a few hits in the database, in accordance with the composite nature of this database. Few $100 \%$ matches were observed. While most environmental sequences were highly similar (93-98\%) to the M. mobilis JLW8 sequences, some sequences diverged as much as $21 \%$ (Table 1). The divergence from the sequences present in the metagenome at the highest coverage (i.e. the most well-assembled sequences) ranged from 0 to $21 \%$. Complete sequences for two proteins, methylene tetrahydromethanopterin dehydrogenase $(\mathrm{MtdB})$ and formaldehyde-activating enzyme (Fae), both involved in formaldehyde metabolism (Chistoserdova et al., 2003), were obtained, and these were compared to the homologues translated from the composite genome in terms of predicted tryptic peptide masses. Only two fullsequence homologues were found for MtdB, both at $93 \%$ amino acid identity (Table 1). Out of the 20 predicted tryptic peptides, $8(40 \%)$ had perfect matches in the database. Four full-sequence homologues were detected for Fae, with amino acid identities ranging from 94 to $98 \%$. Out of the 12 predicted peptides, $10(83 \%)$ had perfect matches in the database. These results suggested that for most proteins, a large portion of the peptides of strain JLW8 obtained by tryptic digestion were expected to have identical matches in the protein database translated from the metagenome.

\section{Qualitative proteome coverage}

Spectral counts as used here are summations of the number of redundant peptides (i.e. including repeated measure- 
ments of the same peptide at different stages of the HPLC separation) observed that map uniquely to a particular protein or protein grouping as defined by the user (Bosch et al., 2008). While the precise relationship between observed spectral counts and abundance within a given proteome is complex, for microbial systems high counts usually correlate with high abundance, as validated by global transcription measurements and other means (Bosch et al., 2008). However, low counts do not necessarily imply low abundance, due to detectability differences among peptides. Data for two biological replicates were collected and analysed. The M. mobilis specific protein database utilized for peptide identification was represented by polypeptide sequences translated from the 12951 gene sequences that constituted the M. mobilis composite genome (covering approximately five closely related M. mobilis genomes; Kalyuzhnaya et al., 2008b), downloaded from the IMG/M database, along with their automated annotations. These annotations were manually curated for proteins with high spectral counts and thus inferred high relative abundance (see Supplementary Table S1, available with the online version of this paper). A total of 2353 and 2336 polypeptides in this database were matched with the peptides identified in the first and the second biological replicate samples, respectively (Table S1), with a positive detection cutoff of at least three peptide matches per protein (at a cutoff of two peptide matches, 2767 and 2787 peptides were identified, respectively; data not shown). This identification rate (approx. $20 \%$ of total proteins in the database) is, as expected, in a lower range compared to the identification rate typical of proteins matched to a database specific for a given organism (up to $60 \%$, for example, in M. extorquens AM1; Bosch et al., 2008), but within the range of the previously conducted metagenome-based proteomic analyses (Ram et al., 2005) and in agreement with the recent models for protein detection efficiency as a function of sequence divergence (Denef et al., 2007). An additional factor affecting the peptide detection was the partial nature of many of the polypeptides translated from the M. mobilis metagenome, due to low sequence assembly (Kalyuzhnaya et al., 2008b).

\section{Protein-based reconstruction of methylotrophy- specific metabolic pathways}

In most cases, peptides of M. mobilis JLW8 were matched to a number of homologous proteins in the database, representing protein clusters, similarly to the results represented in Table 1. Those homologues with positive peptide matches were grouped manually into clusters, and peptide spectral counts within each cluster were summed. Some of the inferred proteins with the highest summed spectral counts observed in both biological replicates were the ones usually found highly expressed in bacteria, such as the translation elongation factor, chaperonins GroEL and DnaK, subunits of ATP synthase, DNA-directed RNA polymerase, ribosomal proteins, etc. (Table S1) that can be defined as core proteins (Callister et al., 2008). However, of most interest to us were the peptides representing major enzymes and pathways specifically related to methylamine metabolism in M. mobilis. One of the most highly expressed metabolic enzymes was hexulosephosphate synthase (Hps, phylotype 1; Table 2, Table S1 and Fig. 1), the first enzyme in the ribulose monophosphate (RMP) cycle operating in formaldehyde assimilation/oxidation in Methylophilaceae (Lidstrom, 2006). The gene for this enzyme is part of a methylotrophy gene island in M. mobilis (Kalyuzhnaya et al., 2008b) that is very similar to an island also found in Methylobacillus flagellatus (Chistoserdova et al., 2007b). Interestingly, both Methylotenera mobilis and Methylobacillus flagellatus encode a second copy of Hps (phylotype 2, approximately 89\% identity with phylotype 1 in M. mobilis). This enzyme is also detectable in the M. mobilis proteome, but at a much lower level of total counts (Table 1). Other enzymes that are parts of the RMP cycle, both assimilatory and dissimilatory branches, were also identified at relatively high spectral counts, the most abundant being transaldolase and transketolase, which are involved in RMP regeneration (Fig. 1). Peptides for the catalytic subunits of methylamine dehydrogenase (MauAB) were found at high counts, along with the proposed novel electronaccepting cytochrome (MauO; Kalyuzhnaya et al., 2008b), suggesting that this is the main primary oxidation system. Interestingly, MauD, whose function has been proposed as being in small-subunit maturation (van der Palen et al., 1997), was found at relatively high counts, unlike the proteins involved in cofactor biosynthesis (for example, MauG; Li et al., 2008), suggesting a possible role as a chaperone. Polypeptides were detected at high counts forming the glutamine synthetase/glutamate synthase (GS/ GOGAT) pathway. While not typically considered as part of the methylamine utilization pathway, the presence of these enzymes suggests a mechanism for utilizing the ammonia that results from methylamine oxidation. In this respect, the incomplete tricarboxylic acid cycle (Kalyuzhnaya et al., 2008b), in addition to its anaplerotic function in providing central metabolism intermediates (Lidstrom, 2006), must play another important role, in providing the $\alpha$-ketoglutarate that is an essential intermediate in the GS/GOGAT cycle. The presence of the activities of methylamine dehydrogenase and the hexulosephosphate synthase/isomerase pair in M. mobilis JLW8 has been previously reported, supporting the analysis conducted here (Kalyuzhnaya et al., 2006). To confirm identification of the GS/GOGAT cycle, we measured the activities of the respective enzymes and found them at high levels ( $95 \pm 3$ and $174 \pm 5 \mathrm{mU}$, respectively). Glutamate dehydrogenase $(\mathrm{GDH})$, another enzyme known to assimilate ammonia, was undetectable in cell extracts, in agreement with the lack of a gene homologue encoding $\mathrm{GDH}$ in the M. mobilis genomic database.

Genes for the tetrahydromethanopterin $\left(\mathrm{H}_{4} \mathrm{MPT}\right)$-linked $\mathrm{C}_{1}$ transfer pathway are found in most methylotrophs (Vorholt et al., 2000; Kalyuzhnaya et al., 2005). In some of 
Table 2. Major enzymes detected in M. mobilis JLW8 with high spectral counts

KDPG, 2-keto-3-deoxy-6-phosphogluconate; MADH, methylamine dehydrogenase; RFAP, ribofuranosylaminobenzene 5'-phosphate.

\begin{tabular}{|c|c|c|c|}
\hline \multirow[t]{2}{*}{ Protein/function } & \multicolumn{2}{|c|}{ Total spectral counts ${ }^{*}$} & \multirow{2}{*}{$\begin{array}{c}\text { Normalized per amino } \\
\text { acid } \dagger\end{array}$} \\
\hline & Replicate 1 & Replicate 2 & \\
\hline Formaldehyde activating enzyme (Fae) & 1434 & 608 & 10.9 \\
\hline Transaldolase (Tal) & 1482 & 1933 & 10.1 \\
\hline Transketolase (Tkt) & 1335 & 967 & 3.5 \\
\hline Enolase (Eno) & 593 & 912 & 3.5 \\
\hline Homologue of formaldehyde-activating enzyme & 308 & 316 & 3.4 \\
\hline Essential for small subunit maturation (MauD) & 203 & 480 & 3.3 \\
\hline Hexulosephosphate synthase (Hps [2)]) & 159 & 503 & 2.9 \\
\hline Ribose 5-phosphate isomerase (Ppi) & 201 & 309 & 2.3 \\
\hline$\beta$-RFAP synthase (MptG) & 147 & 277 & 1.9 \\
\hline Glyceraldehyde phosphate dehydrogenase (Gpd) & 257 & 331 & 1.7 \\
\hline Cytochrome associated with XoxF (XoxG) & 103 & 276 & 1.7 \\
\hline Hexulosephosphate isomerase (Hpi) & 114 & 134 & 1.4 \\
\hline Electron acceptor from MADH (MauO) & 86 & 76 & 1.3 \\
\hline Putative dehydrogenase (XoxF) & 295 & 268 & 0.9 \\
\hline Isocitrate dehydrogenase (Idh) & 220 & 159 & 0.9 \\
\hline PEP synthase (Pps) & 294 & 396 & 0.9 \\
\hline Phosphoglycerate kinase (Pgk) & 135 & 196 & 0.8 \\
\hline MADH small subuinit (MauA) & 59 & 79 & 0.7 \\
\hline Glutamate synthase large subunit (GltA) & 517 & 545 & 0.7 \\
\hline Succinate dehydrogenase $\beta$ subunit (SucD) & 51 & 90 & 0.4 \\
\hline
\end{tabular}

${ }^{\star}$ Number of peptides observed for each entry or group of closely related entries in the metagenomic inferred protein database, including redundant measurements of the same peptide.

$\dagger$ This value is intended as a means for comparison of abundance of proteins of different length.

them, such as Methylobacterium extorquens, this is an essential pathway in both formaldehyde detoxification and energy metabolism (Chistoserdova et al., 1998, 2003), while in others, such as Methylobacillus flagellatus, this pathway has been demonstrated to be non-essential, the cyclic RMP pathway playing the major role (Chistoserdova et al., 2000). Low abundances of most of the $\mathrm{H}_{4}$ MPT-linked enzymes and of the formate dehydrogenases suggest a secondary role for this linear oxidative pathway in Methylotenera mobilis grown on methylamine. However, Fae (formaldehyde-activating enzyme; phylotype 1), the first enzyme in this pathway, was found to be one of the most abundant proteins. The demonstrated function of this enzyme is in directly binding formaldehyde (Vorholt et al., 2000). However, the unusual location of the fae gene in the M. mobilis genome has prompted a hypothesis of its having a secondary role, as a sensor or signal transducer (Kalyuzhnaya et al., 2008b). A second phylotype of Fae (56\% amino acid identity with phylotype $1 \mathrm{Fae}$ ) was also detected in the proteome, at a much lower level. Interestingly, a Fae homologue named Fae2, of unknown function (Chistoserdova et al., 2003; Kalyuzhnaya et al., 2008a), was also observed at high counts, suggesting an important if not yet demonstrated role in $\mathrm{C}_{1}$ metabolism. 


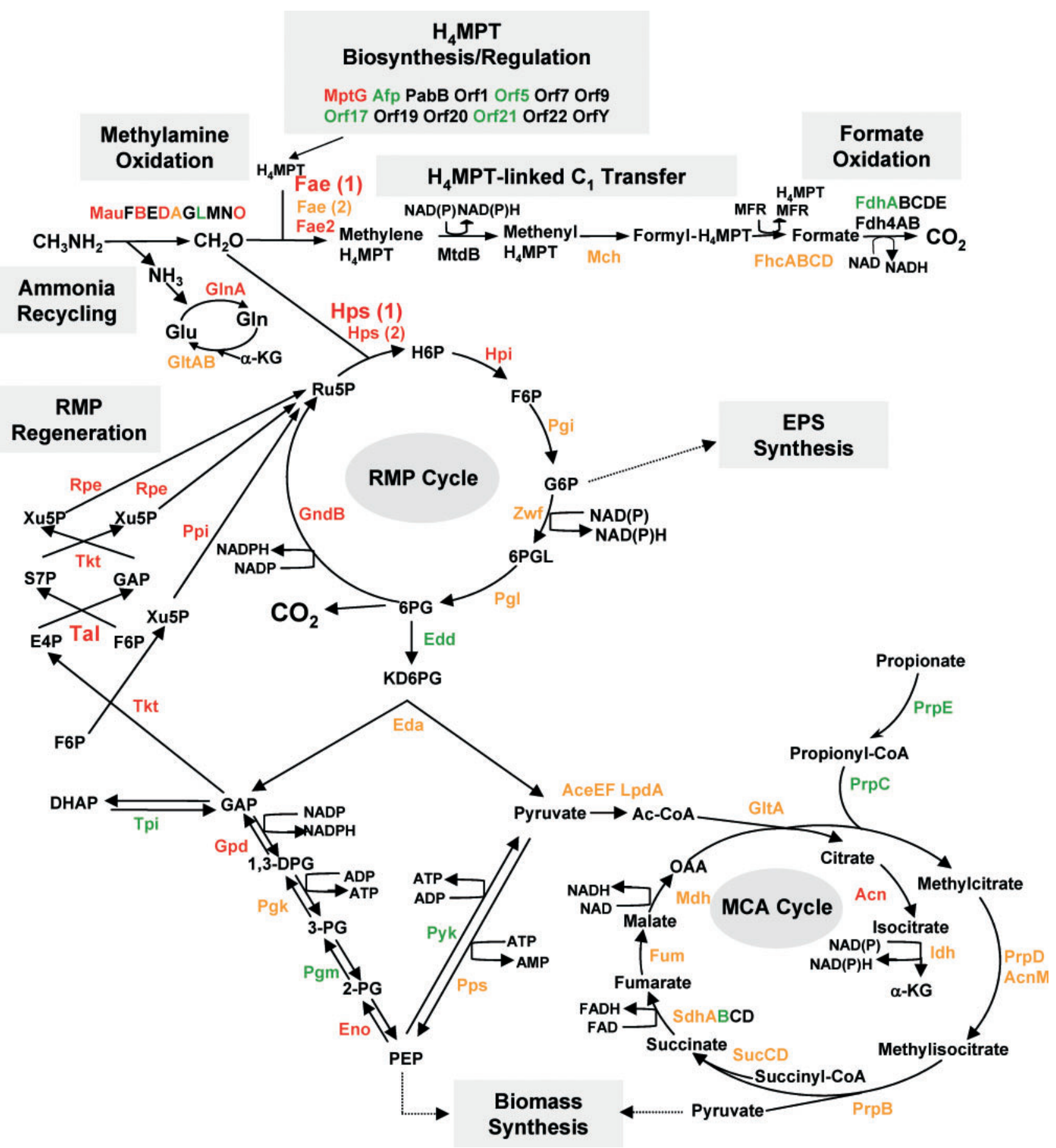

Fig. 1. Central metabolic pathways reconstructed from the composite genome of M. mobilis (Kalyuzhnaya et al., 2008b). Proteins shown in red were found at over 10 peptides normalized per amino acid (see explanation for Table 2; large font) or at over 1 peptide per amino acid (small font). Proteins shown in orange were found at over 0.1 peptide per amino acid, and proteins shown in green were found at less than 0.1 peptides per amino acid. Proteins shown in black were not detected. Enzyme descriptions can be found in Table 1 and in Supplementary Table S1. Abbreviations: $\mathrm{H}_{4} \mathrm{MPT}$, tetrahydromethanopterin; MFR, methanofuran; H6P, hexulose 6-phosphate; F6P, fructose 6-phosphate; G6P, glucose 6-phosphate; 6PGL, 6phosphogluconolactone; 6PG, 6-phosphogluconate; Ru5P, ribulose 5-phosphate; E4P, erythrose 4-phosphate; S7P, sedoheptulose 7-phosphate; Xu5P, xylulose 5-phosphate; GAP, glyceraldehyde phosphate; DHAP, dihydroxyacetone phosphate; DPG, diphosphoglycerate; PG, phosphoglycerate; PEP, phosphoenolpyruvate; Ac-CoA, acetyl-CoA; $\alpha$-KG, $\alpha$ ketoglutarate. 
One metabolic feature that distinguishes Methylotenera mobilis from Methylobacillus flagellatus, as predicted from genome-genome comparisons, is the presence of the genes for a complete methylcitric acid (MCA) cycle. All the proteins constituting this pathway were positively identified in this study (Table 2, Supplementary Table S1 and Fig. 1). The role of this pathway in M. mobilis remains unknown. Its involvement in metabolism of methylamine is not likely as all the essential metabolites are produced in the RMP cycle in combination with the enzymes interconverting $\mathrm{C}_{3}$ metabolites and the partial tricarboxylic acid cycle (Fig. 1). More likely, the presence of the specific MCA cycle proteins during growth on methylamine is due to the peculiarities of regulation of this metabolic pathway. It has been previously suggested that this pathway may play a role in utilization of methylated compounds whose degradation results in propionate as a product (Kalyuzhnaya et al., 2008b). While this hypothesis is a subject for experimental testing, the present study demonstrates expression of the entire set of the genes involved, thus supporting functionality of this pathway.

Curiously, XoxF and XoxG, homologues of, respectively, the large subunit of methanol dehydrogenase and an associated cytochrome, were found at high abundance. The potential role of the Xox system has been attracting attention recently, implicating it in $\mathrm{C}_{1}$ metabolism, but so far without a well-defined function (Wilson et al., 2008; Kalyuzhnaya et al., 2008a). Another highly abundant enzyme was a putative aldehyde dehydrogenase (Adh) whose function also remains unknown.

\section{Conclusions}

We utilized a metagenomic sequence of $M$. mobilis for a strain-specific proteomic analysis of the closely related $M$. mobilis JLW8 grown on methylamine. To address the first specific objective (see Introduction), we provided an experimental validation of sequence assembly and $M$. mobilis-specific sequence binning conducted in our previous study (Kalyuzhnaya et al., 2008b), by detecting proteins involved in major metabolic pathways predicted to operate in metabolism of methylamine. To address the second specific objective, we demonstrated that a metagenomic dataset of inherently lower quality compared to completed genomic sequences is usable for cross-strain protein detection, by identifying a large portion of polypeptides predicted to be encoded in the genome of M. mobilis JLW8, with high confidence. To address the third specific objective, we obtained new insights into the metabolism of methylamine by $M$. mobilis, including expression of multiple pathways for formaldehyde oxidation (linear versus cyclic) and multiple enzyme paralogues (Hps, Fae), suggesting redundancy of key methylotrophy functions previously noted for other organisms (Chistoserdova et al., 2000, 2007a). In addition, we established a potential link between methylamine oxidation and ammonia utilization via the GS/GOGAT pathway. This pathway is likely to play a role in the removal of ammonia produced by the methylamine dehydrogenase reaction to prevent its accumulation to toxic levels. We also demonstrated that all the proteins involved in the proposed MCA cycle were expressed, suggesting a central role for this cycle in M. mobilis metabolism. This work is a prelude to future applications of this metagenomic dataset for protein identification in other M. mobilis strains as well as in natural microbial populations (metagenome-based metaproteomics).

\section{ACKNOWLEDGEMENTS}

This work was funded by the National Science Foundation as part of the Microbial Observatories Program (MCB-0131957). We thank Fred Taub for FileMaker assistance and creation of a proteomic database for M. mobilis, and Mary Lidstrom for helpful comments.

\section{REFERENCES}

Bosch, G., Skovran, E., Xia, Q., Wang, T., Taub, F., Miller, J. A., Lidstrom, M. E. \& Hackett, M. (2008). Comprehensive proteomics of Methylobacterium extorquens AM1 metabolism under single carbon and non-methylotrophic conditions. Proteomics 8, 3494-3505.

Bravo, A. \& Mora, J. (1988). Ammonium assimilation in Rhizobium phaseoli by the glutamine synthetase-glutamate synthase pathway. $J$ Bacteriol 170, 980-984.

Callister, S. J., McCue, L. A., Turse, J. E., Monroe, M. E., Auberry, K. J., Smith, R. D., Adkins, J. N. \& Lipton, M. S. (2008). Comparative bacterial proteomics: analysis of the core genome concept. PLoS One 3, e1542.

Chistoserdova, L., Vorholt, J. A., Thauer, R. K. \& Lidstrom, M. E. (1998). $C_{1}$ transfer enzymes and coenzymes linking methylotrophic bacteria and methanogenic Archaea. Science 281, 99-102.

Chistoserdova, L., Gomelsky, L., Vorholt, J. A., Gomelsky, M., Tsygankov, Y. D., Thauer, R. K. \& Lidstrom, M. E. (2000). Analysis of two formaldehyde oxidation pathways in Methylobacillus flagellatus KT, a ribulose monophosphate cycle methylotroph. Microbiology 146, 233-238.

Chistoserdova, L., Chen, S.-W., Lapidus, A. \& Lidstrom, M. E. (2003). Methylotrophy in Methylobacterium extorquens AM1 from a genomic point of view. J Bacteriol 185, 2980-2987.

Chistoserdova, L., Crowther, G. J., Vorholt, J. A., Skovran, B., Portais, J.-C. \& Lidstrom, M. E. (2007a.). Identification of a fourth formate dehydrogenase in Methylobacterium extorquens AM1 and confirmation of the essential role of formate oxidation in methylotrophy. $J$ Bacteriol 189, 9076-9081.

Chistoserdova, L., Lapidus, A., Han, C., Goodwin, L., Saunders, L., Brettin, T., Tapia, R., Gilna, P., Lucas, S. \& other authors (2007b). The genome of Methylobacillus flagellatus, the molecular basis for obligate methylotrophy, and the polyphyletic origin of methylotrophy. J Bacteriol 189, 4020-4027.

Denef, V. J., Shah, M. B., Verberkmoes, N. C., Hettich, R. L. \& Banfield, J. F. (2007). Implications of strain- and species-level sequence divergence for community and isolate shotgun proteomic analysis. J Proteome Res 6, 3152-3161.

Elias, J. E. \& Gygi, S. P. (2007). Target-decoy search strategy for increased confidence in large-scale protein identifications by mass spectrometry. Nat Methods 4, 207-214. 
Frias-Lopez, J., Shi, Y., Tyson, G. W., Coleman, M. L., Schuster, S. C., Chisholm, S. W. \& DeLong, E. F. (2008). Microbial community gene expression in ocean surface waters. Proc Natl Acad Sci U S A 105, 3805-3810.

Gasteiger, E., Hoogland, C., Gattiker, A., Duvaud, S., Wilkins, M. R., Appel, R. D. \& Bairoch, A. (2005). Protein identification and analysis tools on the ExPASy server. In The Proteomics Protocols Handbook, pp. 571-607. Edited by John M. Walker. Totowa, NJ: Humana Press

Gilbert, J. A., Field, D., Huang, Y., Edwards, R., Li, W., Gilna, P. \& Joint, I. (2008). Detection of large numbers of novel sequences in the metatranscriptomes of complex marine microbial communities. PLoS One 3, e3042.

Kalyuzhnaya, M. G., Korotkova, N., Crowther, G. J., Marx, C. J., Lidstrom, M. \& Chistoserdova, L. (2005). Analysis of gene islands involved in methanopterin-linked $\mathrm{C}_{1}$ transfer reactions reveals new functions and provides evolutionary insights. J Bacteriol 187, 46074614.

Kalyuzhnaya, M. G., Bowerman, S., Lara, J. C., Lidstrom, M. E. \& Chistoserdova, L. (2006). Methylotenera mobilis gen. nov., sp. nov, an obligately methylamine-utilizing bacterium within the family Methylophilaceae. Int J Syst Evol Microbiol 56, 2819-2823.

Kalyuzhnaya, M. G., Hristova, K. R., Lidstrom, M. E. \& Chistoserdova, L. (2008a). Characterization of a novel methanol dehydrogenase in representatives of Burkholderiales: implications for environmental detection of methylotrophy and evidence for convergent evolution. J Bacteriol 190, 3817-3823.

Kalyuzhnaya, M. G., Lapidus, A., Ivanova, N., Copeland, A. C., McHardy, A., Szeto, E., Salamov, A., Grigoriev, I. V., Suciu, D. \& other authors (2008b). High resolution metagenomics targets major functional types in complex microbial communities. Nat Biotechnol 26, 1029-1034.

Konstantinidis, K. T. \& Tiedje, J. M. (2005). Towards a genome-based taxonomy for prokaryotes. J Bacteriol 187, 6258-6264.

Li, X., Fu, R., Liu, A. \& Davidson, V. L. (2008). Kinetic and physical evidence that the diheme enzyme MauG tightly binds to a biosynthetic precursor of methylamine dehydrogenase with incompletely formed tryptophan tryptophylquinone. Biochemistry 47, 2908-2912.

Lidstrom, M. E. (2006). Aerobic methylotrophic prokaryotes. In The Prokaryotes. Edited by A. Balows, H. G. Truper, M. Dworkin, W. Harder \& K.-H. Schleifer. New York: Springer.
Murrell, J. C. \& Dalton, H. (1983). Purification and properties of glutamine synthetase from Methylococcus capsulatus (Bath). J Gen Microbiol 129, 1187-1196.

Ram, R. J., Verberkmoes, N. C., Thelen, M. P., Tyson, G. W., Baker, B. J., Blake, R. C., II, Shah, M., Hettich, R. L. \& Banfield, J. F. (2005). Community proteomics of a natural microbial biofilm. Science 308, 1915-1920.

Rappé, M. S. \& Giovannoni, S. J. (2003). The uncultured microbial majority. Anпu Rev Microbiol 57, 369-394.

Rusch, D. B., Halpern, A. L., Sutton, G., Heidelberg, K. B., Williamson, S., Yooseph, S., Wu, D., Eisen, J. A., Hoffman, J. M. \& other authors (2007). The Sorcerer II Global Ocean Sampling Expedition: Northwest Atlantic through Eastern Tropical Pacific. PLoS Biol 5, e77.

Tringe, S. G., von Mering, C., Kobayashi, A., Salamov, A. A., Chen, A., Chang, H. W., Podar, M., Short, J. M., Mathur, A. J. \& other authors (2005). Comparative metagenomics of microbial communities. Science 308, 554-557.

van der Palen, C. J., Reijnders, W. N., de Vries, S., Duine, J. A. \& Spaning, R. J. (1997). MauE and MauD proteins are essential in methylamine metabolism of Paracoccus denitrificans. Antonie Van Leeuwenhoek 72, 219-228.

Vorholt, J. A., Marx, C. J., Lidstrom, M. E. \& Thauer, R. K. (2000). Novel formaldehyde-activating enzyme in Methylobacterium extorquens AM1 required for growth on methanol. J Bacteriol 182, 66456650.

Wilson, S. M., Gleisten, M. P. \& Donohue, T. J. (2008). Identification of proteins involved in formaldehyde metabolism by Rhodobacter sphaeroides. Microbiology 154, 296-305.

Xia, Q., Wang, T., Taub, F., Park, Y., Capestany, C. A., Lamont, R. J. \& Hackett, M. (2007a). Quantitative proteomics of intracellular Porphyromonas gingivalis. Proteomics 7, 4323-4337.

Xia, Q., Wang, T., Park, Y., Lamont, R. J. \& Hackett, M. (2007b). Differential quantitative proteomics of Porphyromonas gingivalis by linear ion trap mass spectrometry: non-label methods comparison, $q$ values and LOWESS curve fitting. Int J Mass Spectrom 259, 105-116.

Yarrison, G., Young, D. W. \& Choules, G. L. (1972). Glutamate dehydrogenase from Mycoplasma laidlawii. J Bacteriol 110, 494-503.

Edited by: J. A. Vorholt 